\title{
Letter to editor: Evaluation of the relationship between TREM- 1/TREM- 2 ratio and clinical course in COVID- 19 pneumonia
}

\author{
zhihong wang ${ }^{1}$ \\ ${ }^{1}$ Huzhou Central Hospital
}

September 24, 2021

\begin{abstract}
Dear editor, we read with great interest the well written article by Dr Kerget et al with the main objective of investigating the role of TREM-1/TREM-2 ratio on patients with COVID-19 pneumonia. The article pointed that TREM-1 and TREM-2 have important role in inflammation and TREM-1/TREM-2 ratio was higher in severe COVID-19 patients compared with moderate COVID-19 patients. We have certain comments to understand the conclusions of this article. Firstly, triggering receptor expressed on myeloid cells-1 (TREM-1) is mainly express on neutrophils and monocytes in a cell membrane-bound form. A soluble form of TREM-1(sTREM-1), which lacks the cytoplasmic tail and transmembrane part, were detected in the blood in recent studies. Since you have mentioned "serum TREM-1", we were confused whether you detected TREM-1 or sTREM-1. Secondly, we wanted to know more about the treatment and the kidney functions of the patients. Thirdly, We are curious to see if high TREM-1/TREM-2 ratio could predict the distribution of ILD. We would be glad to hear the opinion of the author on the points, to get a more convincing conclusion.
\end{abstract}

Letter to editor: Evaluation of the relationship between TREM-1/TREM-2 ratio and clinical course in COVID-19 pneumonia

Zhihong Wang

Department of Rheumatology and Immunology, Huzhou Central Hospital, Affiliated Central Hospital of Huzhou Normal University, Huzhou, 313003, People's Republic of China.

\section{Correspondence:}

Zhihong Wang

Department of Rheumatology and Immunology, Huzhou Central Hospital, Affiliated Central Hospital of Huzhou Normal University, Huzhou, 313003, People's Republic of China, E-mail:zhihong1017@126.com

Dear editor, we read with great interest the well written article by Dr Kerget et al with the main objective of investigating the role of TREM-1/TREM-2 ratio on patients with COVID-19 pneumonia ${ }^{[1]}$. The article pointed that TREM-1 and TREM-2 have important role in inflammation and TREM-1/TREM-2 ratio was higher in severe COVID-19 patients compared with moderate COVID-19 patients. We have certain comments to understand the conclusions of this article.

To begin with the measurement of biochemical markers, triggering receptor expressed on myeloid cells-1 (TREM-1) is mainly express on neutrophils and monocytes in a cell membrane-bound form. A soluble form of TREM-1(sTREM-1), which lacks the cytoplasmic tail and transmembrane part, was detected in the blood in recent studies. Since the authors mentioned "serum TREM-1", we were confused whether they detected TREM-1 or sTREM-1. Regarding the demographic features, we wanted to know more about the treatment 
and the kidney functions of the patients. As we all known, treatment could affect the serum levels of sTREM1 or other inflammatory cytokines. Besides, it has been reported that TREM-1 seems to be correlated with renal function and acute kidney injury following COVID-19 infection has been described in recent literature. Lastly, according to a previous report ${ }^{[2]}$, lesions could be assessed in four lung zones and the extent of ILD lesions was semi-quantitatively scored based on the percentage of the lung parenchyma involved in each zone. The COVID-19 pandemic is characterized by an interstitial pneumonia. We are curious to see if high TREM-1/TREM-2 ratio could predict the distribution of ILD. Furthermore, we wanted to know if TREM1/TREM-2 ratio in urine might be correlated with clinical course in COVID-19 pneumonia since urine was obtained in this article.

We would be glad to hear the opinion of the author on the points, to get a more convincing conclusion.

Disclosure statement

The authors have declared no conflicts of interest for this article.

Data availability statement

None

Reference:

[1] KERGET F, KERGET B, IBA YıLMAZ S, et al. Evaluation of the relationship between TREM1/TREM-2 ratio and clinical course in COVID-19 pneumonia [J]. International journal of clinical practice, 2021, e14697.

[2] GUNNARSSON R, AALøKKEN T M, MOLBERG $\varnothing$, et al. Prevalence and severity of interstitial lung disease in mixed connective tissue disease: a nationwide, cross-sectional study [J]. Annals of the rheumatic diseases, 2012, 71(12): 1966-72. 